MITH-97/10

\title{
Further discussions on a possible lattice chiral gauge theory
}

\author{
She-Sheng Xue ${ }^{(a)}$ \\ INFN - Section of Milan, Via Celoria 16, Milan, Italy
}

\begin{abstract}
In a possible $S U_{L}(2)$ lattice chiral gauge theory with a large multifermion coupling, we try to further clarify the threshold phenomenon: the possibility that the right-handed three-fermion state turns into the virtual states of its constituents (free chiral fermions) in the low-energy limit. Provided this phenomenon occurs, we discuss the chiral gauge coupling, Ward identities and the gauge anomaly within the gauge-invariant prescription of the perturbative chiral gauge theory.
\end{abstract}

June, 1996

PACS 11.15Ha, 11.30.Rd, 11.30.Qc

a) E-mail address: xue@milano.infn.it 
1. Since the "no-go" theorem [1] of Nielsen and Ninomiya was demonstrated in 1981, the problem of chiral fermion "doubling" and "vector-like" feature on a lattice still exists if one insists on preserving chiral gauge symmetries. Actually, the essential spirit of the "no-go" theorem of Nielsen and Ninomiya is that, under certain prerequisites, the paradox concerning chiral gauge symmetries, vector-like doubling and anomalies are unavoidable.

Eichten and Preskill [2] proposed a model of multifermion couplings ten years ago. The crucial points of multifermion coupling can be briefly described as follows. Multifermion couplings are introduced such that, in the strong-coupling phase, Weyl states composing three elementary Weyl fermions (three-fermion states) are bound. Then, these three-fermion states pair up with elementary Weyl fermions to become Dirac fermions. Such Dirac fermions may be massive without violating chiral gauge symmetries, due to the appropriate quantum numbers and chirality carried by these three-fermion states. The binding thresholds of such three-fermion states depend on elementary Weyl modes residing in different regions of the Brillouin zone. If one assumes that the spontaneous symmetry breaking of the Nambu-Jona Lasinio (NJL) type [3] does not occur and such binding thresholds separate the weak-coupling symmetric phase from the strongcoupling symmetric phase, there are two possibilities to realize the continuum limit of chiral fermions in coupling space. One is to cross the binding threshold of the three-fermion state of chiral fermions; another is of a wedge between two thresholds, where the three-fermion state of chiral fermions has not been formed, provided all doublers sitting in various edges of the Brillouin zone have been bound to be massive Dirac fermions and decouple.

The model[2] was studied in ref. [4], where it was pointed out that such a model of multifermion couplings fails to give chiral fermions in the continuum limit. The reasons are that an NJL spontaneous symmetry-breaking phase separating the strong-coupling symmetric phase from the weak-coupling symmetric phase, the right-handed Weyl states do not completely disassociate from the left-handed chiral fermions and the phase structure of such a model of multifermion couplings is similar to that of the Smit-Swift (Wilson-Yukawa) model[5], which has been very carefully studied and shown to fail [6].

We should not be surprised that a particular class of multifermion couplings or corresponding Yukawa coupling models does not work. This does not means that all possible classes of multifermion couplings or corresponding Yukawa coupling models definitely do not work. In the ref. [7], we studied a possible lattice chiral theory with an extremely large multifermion coupling $\left(g_{1}=0,1 \ll g_{2}<\infty\right)$ :

$$
\begin{aligned}
S & =\frac{1}{2 a} \sum_{x}\left(\bar{\psi}_{L}^{i}(x) \gamma_{\mu} D^{\mu} \psi_{L}^{i}(x)+\bar{\psi}_{R}(x) \gamma_{\mu} \partial^{\mu} \psi_{R}(x)\right) \\
& +g_{2} \bar{\psi}_{L}^{i}(x) \cdot \Delta \psi_{R}(x) \Delta \bar{\psi}_{R}(x) \cdot \psi_{L}^{i}(x) .
\end{aligned}
$$

This action possesses the $\psi_{R}$ shift-symmetry 8 . The important consequences of the Ward identity of this symmetry are that [7], for any finite values of the 
multifermion coupling $g_{2}$, the normal modes $(p \rightarrow 0)$ of $\psi_{L}^{i}(x)$ and $\psi_{R}$ do not undergo NJL spontaneous symmetry breaking for vanishing self-energy functions,

$$
p_{\mu}=0, \quad \Sigma^{i}(p)=0,
$$

and do not bind to three-fermion states in the continuum limit. It is due to these properties that we have the possibility of finding a segment[7]

$$
1 \ll g_{2}<\infty,
$$

where normal modes remain as free chiral fermions, only doublers $\left(p \simeq \pi_{A}\right)$ of $\psi_{L}^{i}$ and $\psi_{R}$ in (1) are bound to form three-fermion states:

$$
\Psi_{R}^{i}=\frac{1}{2 a}\left(\bar{\psi}_{R} \cdot \psi_{L}^{i}\right) \psi_{R} ; \quad \Psi_{L}^{n}=\frac{1}{2 a}\left(\bar{\psi}_{L}^{i} \cdot \psi_{R}\right) \psi_{L}^{i} .
$$

These three-fermion states carry the appropriate quantum numbers of the chiral gauge group $S U_{L}(2)$ that accommodates the $\psi_{L}^{i}$. These bound states (4) are Weyl fermions and respectively pair up with the $\bar{\psi}_{R}$ and $\bar{\psi}_{L}^{i}$ to be massive, neutral $\Psi_{n}$ and charged $\Psi_{c}^{i}$ Dirac fermions,

$$
\Psi_{c}^{i}=\left(\psi_{L}^{i}, \Psi_{R}^{i}\right), \quad \Psi_{n}=\left(\Psi_{L}^{n}, \psi_{R}\right) .
$$

And their inverse propagators are:

$$
\begin{aligned}
S_{c}^{-1}(p)_{i j} & =\delta_{i j}\left(\frac{i}{a} \sum_{\mu} \gamma_{\mu} \sin p^{\mu} P_{L}+\frac{i}{a} \sum_{\mu} \gamma_{\mu} \sin p^{\mu} P_{R}+M(p)\right), \\
S_{n}^{-1}(p) & =\sum_{\mu} \gamma_{\mu} f^{\mu}(p) P_{L}+\frac{i}{a} \sum_{\mu} \gamma_{\mu} \sin p^{\mu} P_{R}+M(p) .
\end{aligned}
$$

The doubler spectrum of the massive composite Dirac fermions $\Psi_{c}^{i}$ and $\Psi_{n}$ (5) is vector-like, consistently with the $S U_{L}(2) \otimes U_{R}(1)$ chiral symmetry. This segment (37) gives us a loophole to have chiral fermions in the continuum limit.

In the present letter, in order to be more clear that normal modes could remain as free chiral fermions, we further clarify the threshold phenomenon: the possibility of three-fermion states turning into the virtual states of their constituents in the low-energy limit. Provided this phenomenon occurs, we discuss the perturbation of an $S U_{L}(2)$ chiral gauge interaction and gauge anomaly.

2. We discuss how the normal modes $(p=\tilde{p} \sim 0)$ of the $\psi_{L}^{i}(x)$ and $\psi_{R}(x)$ are possibly massless and chiral in the low-energy limit. This is most difficult point to show for the time being, since the strong-coupling expansion in powers of $\frac{1}{g_{2}}$ breaks down for $p \rightarrow 0$ [7]. On the basis of the continuity [1, 9] of the vector-like spectrum (6.7) of doublers in momentum space due to the locality of the theory (11), one may argue that the vector-like spectrum (7.66), which is obtained for 
$p \neq 0$, can be continuously extrapolated to $p \rightarrow 0$, and we fail to have chiral fermions in the low-energy region.

Whether the spectrum of normal modes $(p \rightarrow 0)$ of $\psi_{L}^{i}$ and $\psi_{R}$ is chiral, is crucially related to, whether the normal modes have been composed to form the three-fermion states (5) in the segment $1 \ll g_{2}<\infty$. As $p \rightarrow 0$, the effective multifermion coupling for these normal modes becomes small, as does the binding energy of these three-fermion states. The continuity of the spectrum (70,6) in momentum space breaks down at the threshold where the binding energy of these three-fermion states goes to zero. In the following discussion, we adopt the $1+1$ dimensional case to illustrate this threshold phenomenon 10.

We take the charged Dirac fermion (6) on its mass shell and consider that the time direction is continuous and one space direction is discrete. We obtain the dispersion relation corresponding to this Dirac fermion (6) for $p \neq 0$,

$$
E(p)= \pm \sqrt{\sin ^{2} p+\left(8 a^{2} g w^{2}(p)\right)^{2}}
$$

where $E(p)$ is the dimensionless energy of the state " $p$ ". In eq.(8), the "+" sign corresponds to the dispersion relation of the right-handed three-fermion state $\Psi_{R}^{i}(x)$. Due to the locality of the theory, the spectrum of this bound state $\Psi_{R}^{i}(x)$ is continuous in momentum space [1, 9]. The vector-like spectrum (8) that we obtained by the strong coupling expansion at $p \sim \mathrm{O}(1)$, can be analytically continued to low-momentum states $p \rightarrow 0$, unless this bound state $\Psi_{R}^{i}$ hits the energy threshold for dissolving into its constituents.

For a given total momentum $p$ in the low-energy region, we consider a fermion system that contains the same constituents of the bound state $\Psi_{R}^{i}(x)$. This fermion system is the virtual state of three free chiral fermions: right-handed fermions $\bar{\psi}_{R}$ and $\psi_{R}$ with momenta $p_{1}$ and $p_{2}$, and a left-handed fermion $\psi_{L}^{i}$ with momentum $p_{3}$, where

$$
p=p_{1}+p_{2}+p_{3}>0, \quad\left|p_{i}\right| \ll \frac{\pi}{2}, \quad i=1,2,3 .
$$

Since the NJL spontaneous symmetry breaking does not occur (2) for the states $\left|p_{i}\right| \rightarrow 0$ in the segment $\left(1 \ll g_{2}<\infty\right)$, the total energy (continuous spectrum) of such a system is given by

$$
\begin{aligned}
E_{t} & =E_{1}\left(p_{1}\right)+E_{2}\left(p_{2}\right)+E_{3}\left(p_{3}\right), \\
E_{1}\left(p_{1}\right) & =\sqrt{\sin ^{2} p_{1}}, \quad p_{1}>0, \\
E_{2}\left(p_{2}\right) & =\sqrt{\sin ^{2} p_{2}}, \quad p_{2}>0, \\
E_{3}\left(p_{3}\right) & =-\sqrt{\sin ^{2} p_{3}}, \quad p_{3}<0,
\end{aligned}
$$

where all negative-energy states have been filled. There is no definite relationship between the total energy $E_{t}$ and the total momentum $p$, since this system is not a 
particle (a bound state). The total energy $E_{t}$ of such a virtual state is continuous because of the relative degrees of freedom $\left(p_{1}, p_{2}, p_{3}\right)$ within the system.

Given the total momentum $p$, the lowest energy min $E_{t}$ (the threshold) and corresponding configuration $\left(p_{1}, p_{2}, p_{3}\right)$ of such a virtual state can be determined by minimizing the following total energy with a Legendre multiplier $\lambda$ (constraint (9))

$$
E_{t}=E_{1}\left(p_{1}\right)+E_{2}\left(p_{2}\right)+E_{3}\left(p_{3}\right)+\lambda p
$$

We obtain

$$
\begin{aligned}
\min E_{t}(p) & =3|\sin p|, \\
p_{3} & =-p, \quad p_{1}=p_{2}=p .
\end{aligned}
$$

On the other hand, the three-fermion state (5), as a bound state, has a definite relationship between its energy and momentum, which is given by the dispersion relation (8) with the "+" sign. Given the same momentum " $p$ " as eq.(9), this bound state is stable, if and only if there is an energy gap $\delta(p)$ (binding energy) between the threshold (12) and the energy (8) of the three-fermion state, i.e.,

$$
\delta(p)=\min E_{t}(p)-E(p)>0 .
$$

The three-fermion state dissolves into its constituents, when the energy gap $\delta$ goes to zero,

$$
\delta(p)=\min E_{t}(p)-E(p)=0 .
$$

The same discussion can be applied to the neutral three-fermion state $\Psi_{L}^{n}$ (7). This discussion is very much like the case of the hydrogen atom, a bound state composed of an electron and a proton, where the energy gap between the first energy-level $(n=1)$ and the continuous spectrum of its virtual state is $13.6 \mathrm{eV}$. The hydrogen atom turns into the virtual state of a free electron and a free proton as the energy-gap disappears $(n \gg 1)$.

Substituting eqs.(8) and (12) into eq.(14), we obtain in the continuum limit $p \rightarrow 0$, the energy-gap closes,

$$
\delta(p) \rightarrow 0, \quad p \rightarrow 0
$$

where the three-fermion-state dissolves into the virtual state of three free chiral fermions. Obviously, this plausible speculation needs to receive either a rigorously analytical proof or a numerical evidence, which are the subject of future work. Nevertheless, We assume there exist a threshold $\epsilon$ in momentum space. The low-energy fermion states " $p$ " below this threshold $\epsilon$

$$
|p|<\epsilon \ll \frac{\pi}{2}
$$

are massless and chiral. This threshold $\epsilon$ certainly depends on the multifermion coupling $g_{2}$. 
To end this discussion, we would like to point out that the fact that the normal modes do not undergo NJL spontaneous symmetry breaking (2) for any finite value of the multifermion coupling $g_{2}$ is extremely crucial. This means, with respect to normal modes, there is no broken phase separating the strong symmetric phase from the weak symmetric phase. In other words, there is no a mass-gap in eq. (10). Otherwise, the system (10) would be massive, the energy gap (13) would never be zero and we would end up with a vector-like spectrum in the low-energy region $(p \rightarrow 0)$. This is the main reason for the failure of EP's model, as pointed out in ref. [4. Thus, there might be a chance that (i) the chiral continuum limit can be defined at a transition from one symmetric phase to another symmetric phase; (ii) there is a region (3) in the coupling space $g_{2}$ where doublers are gauge-invariantly decoupled and normal modes are chiral (non NJLgenerated masses). The latter is a possible case in the segment $\left(1 \ll g_{2}<\infty\right)$, which we have discovered.

3. Can the scaling region $1 \ll g_{2}<\infty$ be altered, as the $S U_{L}(2)$ chiral gauge coupling $g$ is turned on in the action (1)? We expect a slight change of the scaling segment. We should be able to re-tune the multifermion coupling $g_{2}$ to compensate these changes, due the fact that the $S U_{L}(2)$-chiral gauge interaction does not spoil the $\psi_{R}$ shift-symmetry. As a consequence, the Ward identity associated with the $\psi_{R}$ shift-symmetry remains valid when the chiral gauge interaction is turned on.

Based on the Ward identity of the $\psi_{R}$ shift-symmetry (15) in ref.[7], we take functional derivatives with respect to the gauge field $A_{\mu}^{\prime}$, and we arrive at the following Ward identities,

$$
\frac{\delta^{(2)} \Gamma}{\delta A_{\mu}^{\prime} \delta \bar{\psi}_{R}^{\prime}}=\frac{\delta^{(3)} \Gamma}{\delta A_{\mu}^{\prime} \delta \psi_{R}^{\prime} \delta \bar{\psi}_{R}^{\prime}}=\frac{\delta^{(3)} \Gamma}{\delta A_{\mu}^{\prime} \delta \Psi_{L}^{\prime n} \delta \bar{\psi}_{R}^{\prime}}=\cdots=0 .
$$

These Ward identities and the identical vanishing of 1PI functions containing external gauge fields $A_{\mu}(x)$, "spectator" fermion $\psi_{R}(x)$ and neutral composite field $\Psi_{L}^{n}(x)$ show non interaction between the gauge field and "spectator" fermion $\psi_{R}$ and neutral three-fermion states $\Psi_{L}^{n}(x)$. Thus, we disregard these neutral modes.

In order to find the interacting vertex between the gauge boson and the charged Dirac fermion $\Psi_{c}^{i}(x)$, we need to consider the following three-point functions,

$$
\begin{aligned}
\left\langle\Psi_{c}\left(x_{1}\right) \bar{\Psi}_{c}(x) A_{\nu}^{a}(y)\right\rangle & =\left\langle\psi_{L}\left(x_{1}\right) \bar{\psi}_{L}(x) A_{\nu}^{a}(y)\right\rangle+\left\langle\psi_{L}\left(x_{1}\right) \bar{\Psi}_{R}(x) A_{\nu}^{a}(y)\right\rangle \\
& +\left\langle\Psi_{R}\left(x_{1}\right) \bar{\psi}_{L}(x) A_{\nu}^{a}(y)\right\rangle+\left\langle\Psi_{R}\left(x_{1}\right) \bar{\Psi}_{R}(x) A_{\nu}^{a}(y)\right\rangle
\end{aligned}
$$

where we omit henceforth the $S U_{L}(2)$ indices $i$ and $j$. Assuming the vertex functions to be $\Lambda_{\mu}^{a}\left(p, p^{\prime}\right)$ and $q=p^{\prime}+p$, we can write the three-point functions 
in momentum space:

$$
\begin{aligned}
\int_{x_{1} x y} e^{i\left(p^{\prime} x+p x_{1}-q y\right)}\left\langle\psi_{L}\left(x_{1}\right) \bar{\psi}_{L}(x) A_{\nu}^{a}(y)\right\rangle & =G_{\nu \mu}^{a b}(q) S_{L L}(p) \Lambda_{\mu L L}^{b}\left(p, p^{\prime}\right) S_{L L}\left(p^{\prime}\right) ;(19) \\
\int_{x_{1} x y} e^{i\left(p^{\prime} x+p x_{1}-q y\right)}\left\langle\psi_{L}\left(x_{1}\right) \bar{\Psi}_{R}(x) A_{\nu}^{a}(y)\right\rangle & =G_{\nu \mu}^{a b}(q) S_{L L}(p) \Lambda_{\mu L R}^{b}\left(p, p^{\prime}\right) S_{R R}\left(p^{\prime}\right)(20) \\
\int_{x_{1} x y} e^{i\left(p^{\prime} x+p x_{1}-q y\right)}\left\langle\Psi_{R}\left(x_{1}\right) \bar{\Psi}_{R}(x) A_{\nu}^{a}(y)\right\rangle & =G_{\nu \mu}^{a b}(q) S_{R R}(p) \Lambda_{\mu R R}^{b}\left(p, p^{\prime}\right) S_{R R}\left(p^{\prime}\right)(21) \\
\int_{x_{1} x y} e^{i\left(p^{\prime} x+p x_{1}-q y\right)}\left\langle\Psi_{c}\left(x_{1}\right) \bar{\Psi}_{c}(x) A_{\nu}^{a}(y)\right\rangle & =G_{\nu \mu}^{a b}(q) S_{c}(p) \Lambda_{\mu c}^{b}\left(p, p^{\prime}\right) S_{c}\left(p^{\prime}\right),
\end{aligned}
$$

where $G_{\nu \mu}^{a b}(q)$ is the propagator of the gauge boson; $S_{L L}(p), S_{R R}(p)$ and $S_{c}(p)$ are the propagators of chiral fermions $\psi_{L}(x), \Psi_{R}(x)$ and Dirac fermion $\Psi_{c}(x)$.

Using the small gauge-coupling expansion, one can directly calculate the three-point function:

$$
\begin{aligned}
& \left\langle\psi_{L}\left(x_{1}\right) \bar{\psi}_{L}(x) A_{\mu}^{a}(y)\right\rangle=i \frac{g}{2}\left(\frac{\tau^{a}}{2}\right) \sum_{z}\left\langle\psi_{L}\left(x_{1}\right) \bar{\psi}_{L}(x)\right\rangle \gamma_{\rho} \\
& {\left[\left\langle\psi_{L}(z+\rho) \bar{\psi}_{L}(x)\right\rangle\left\langle A_{\rho}^{b}\left(z+\frac{\rho}{2}\right) A_{\mu}^{a}(y)\right\rangle+\left\langle\psi_{L}(z-\rho) \bar{\psi}_{L}(x)\right\rangle\left\langle A_{\rho}^{b}\left(z-\frac{\rho}{2}\right) A_{\mu}^{a}(y)\right\rangle\right](23)}
\end{aligned}
$$

and obtain

$$
\begin{aligned}
\Lambda_{\mu L L}^{(1)}\left(p, p^{\prime}\right) & =i g\left(\frac{\tau^{a}}{2}\right) \cos \left(\frac{p+p^{\prime}}{2}\right)_{\mu} \gamma_{\mu} P_{L} \\
\Lambda_{\mu \nu L L}^{(2)}\left(p, p^{\prime}\right) & =-i \frac{g^{2}}{2}\left(\frac{\tau^{a} \tau^{b}}{4}\right) \sin \left(\frac{p+p^{\prime}}{2}\right)_{\mu} \gamma_{\mu} \delta_{\mu \nu} P_{L} \\
& \ldots
\end{aligned}
$$

By the strong coupling expansion in powers of $\frac{1}{g_{2}}$, we try to compute the other three-point functions in eqs.(18) in terms of $\left\langle\psi_{L}\left(x_{1}\right) \bar{\psi}_{L}(x) A_{\nu}^{a}(y)\right\rangle$, and we obtain the following recursion relations at the leading nontrivial order,

$$
\begin{aligned}
\left\langle\psi_{L}\left(x_{1}\right) \bar{\psi}_{L}(x) A_{\nu}^{a}(y)\right\rangle & =\frac{1}{g_{2} \Delta^{2}(x)}\left(\frac{1}{2 a}\right)^{2} \sum_{\rho}^{\dagger}\left\langle\psi_{L}\left(x_{1}\right) \bar{\Psi}_{R}(x+\rho) A_{\nu}^{a}(y)\right\rangle \gamma_{\rho} \\
\left\langle\psi_{L}\left(x_{1}\right) \bar{\psi}_{L}(x) A_{\nu}^{a}(y)\right\rangle & =\frac{1}{g_{2} \Delta^{2}\left(x_{1}\right)}\left(\frac{1}{2 a}\right)^{2} \sum_{\rho}^{\dagger} \gamma_{\rho}\left\langle\Psi_{R}\left(x_{1}+\rho\right) \bar{\psi}_{L}(x) A_{\nu}^{a}(y)\right\rangle \\
\left\langle\Psi_{R}\left(x_{1}\right) \bar{\Psi}_{R}(x) A_{\nu}^{a}(y)\right\rangle & =\frac{1}{g_{2} \Delta^{2}(x)}\left(\frac{1}{2 a}\right)^{2} \sum_{\rho}^{\dagger} \gamma_{\rho}\left\langle\psi_{L}\left(x_{1}\right) \bar{\Psi}_{R}(x+\rho) A_{\nu}^{a}(y)\right\rangle .
\end{aligned}
$$

We make the Fourier transform in both sides of the above recursion relations for $\left(p, p^{\prime} \neq 0\right)$, and obtain,

$$
S_{L L}(p) \Lambda_{\mu L L}^{a}\left(p, p^{\prime}\right) S_{L L}\left(p^{\prime}\right)=\frac{i}{a M\left(p^{\prime}\right)} S_{L L}(p) \Lambda_{\mu L R}^{a}\left(p, p^{\prime}\right) S_{R R}\left(p^{\prime}\right) \sum_{\rho} \sin p_{\rho}^{\prime} \gamma^{\oplus} 2
$$




$$
\begin{aligned}
S_{L L}(p) \Lambda_{\mu L L}^{a}\left(p, p^{\prime}\right) S_{L L}\left(p^{\prime}\right) & =\frac{i}{a M(p)} \sum_{\rho} \sin p_{\rho} \gamma^{\rho} S_{R R}(p) \Lambda_{\mu R L}^{a}\left(p, p^{\prime}\right) S_{L L}\left(p^{\prime}\right)(29) \\
S_{R R}(p) \Lambda_{\mu R R}^{a}\left(p, p^{\prime}\right) S_{R R}\left(p^{\prime}\right) & =\frac{i}{a M\left(p^{\prime}\right)} \sum_{\rho} \sin p_{\rho}^{\prime} \gamma^{\rho} S_{L L}(p) \Lambda_{\mu L R}^{a}\left(p, p^{\prime}\right) S_{R R}\left(p^{\prime} \gamma^{\prime} 30\right)
\end{aligned}
$$

In these equations, the propagator of the gauge boson, $G_{\nu \mu}^{a b}(q)$, is eliminated from both sides of equations.

Using these recursion relations (28-30) and $S_{L L}(p), S_{R R}(p)$ obtained in ref. [7], we can compute the vertex functions $\Lambda_{\mu R L}^{a}\left(p, p^{\prime}\right), \Lambda_{\mu L R}^{a}\left(p, p^{\prime}\right)$ and $\Lambda_{\mu R R}^{a}\left(p, p^{\prime}\right)$ in terms of the vertex function $\Lambda_{\mu L L}^{a}\left(p, p^{\prime}\right)$ (24),

$$
\begin{aligned}
M\left(p^{\prime}\right) \Lambda_{\mu L L}^{a}\left(p, p^{\prime}\right) & =\Lambda_{\mu L R}^{a}\left(p, p^{\prime}\right)\left(\frac{i}{a}\right) \sum_{\rho} \sin p_{\rho}^{\prime} \gamma^{\rho} \\
M(p) \Lambda_{\mu L L}^{a}\left(p, p^{\prime}\right) & =\left(\frac{i}{a}\right) \sum_{\rho} \sin p_{\rho} \gamma^{\rho} \Lambda_{\mu R L}^{a}\left(p, p^{\prime}\right) \\
M\left(p^{\prime}\right) \Lambda_{\mu R R}^{a}\left(p, p^{\prime}\right) & =\left(\frac{i}{a}\right) \sum_{\rho} \sin p_{\rho}^{\prime} \gamma^{\rho} \Lambda_{\mu L R}^{a}\left(p, p^{\prime}\right)
\end{aligned}
$$

Taking $\Lambda_{\mu L L}^{a}\left(p, p^{\prime}\right)$ to be eq.(24) at leading order, we obtain

$$
\begin{aligned}
\Lambda_{\mu R R}^{(1)}\left(p, p^{\prime}\right) & =i g\left(\frac{\tau^{a}}{2}\right) \cos \left(\frac{p+p^{\prime}}{2}\right)_{\mu} \gamma_{\mu} P_{R}, \\
\Lambda_{\mu L R}^{(1)}\left(p, p^{\prime}\right)\left(\frac{i}{a}\right) \sin p_{\mu}^{\prime} & =\frac{1}{2} M\left(p^{\prime}\right) i g\left(\frac{\tau^{a}}{2}\right) \cos \left(\frac{p+p^{\prime}}{2}\right)_{\mu}, \\
\left(\frac{i}{a}\right) \sin p_{\mu} \Lambda_{\mu R L}^{(1)}\left(p, p^{\prime}\right) & =\frac{1}{2} M(p) i g\left(\frac{\tau^{a}}{2}\right) \cos \left(\frac{p+p^{\prime}}{2}\right)_{\mu} .
\end{aligned}
$$

Thus, the coupling (18) between the gauge field and Dirac fermion (6) is given by

$$
\Lambda_{\mu c}^{(1)}=\Lambda_{\mu L L}^{(1)}+\Lambda_{\mu L R}^{(1)}+\Lambda_{\mu R L}^{(1)}+\Lambda_{\mu R R}^{(1)}
$$

These calculations can be straightforwardly generalized to higher orders of the perturbative expansion in powers of the gauge coupling. One can check that these results precisely obey the following Ward identity of the exact $S U_{L}(2)$ chiral gauge symmetry $\left(p^{\prime}, p \neq 0\right)$,

$$
\left(\frac{i}{a}\right)\left(\sin p_{\mu}-\sin p_{\mu}^{\prime}\right) \Lambda_{\mu c}^{(1)}\left(p, p^{\prime}\right)=S_{c}^{-1}(p)-S_{c}^{-1}\left(p^{\prime}\right),
$$

where the gauge coupling $g$ and generator $\frac{\tau_{a}}{2}$ are eliminated from the vertex $\Lambda_{\mu c}$. These results are what we expected, since we are in the symmetric phase $\left(1 \ll g_{2}<\infty\right)$ where the exact $S U_{L}(2)$ chiral gauge symmetry is realized by the vector-like spectrum excluding the low-energy states $p^{\prime} \neq 0$ and $p \neq 0$. 
When the momenta $p^{\prime}, p$ (38) reach the threshold $\epsilon$ (16), the right-handed three-fermion state $\Psi_{R}^{i}(x)$ is supposed to dissolve into its constituents. The 1PI vertex functions $\Lambda_{\mu R R}, \Lambda_{\mu R L}$, and $\Lambda_{\mu L R}$ relating to $\Psi_{R}^{i}(x)$ vanish at this threshold. The coupling vertex (37) between the gauge boson and fermion should turn out to be chiral consistently with the $S U_{L}(2)$ chiral gauge symmetry, $\left(p^{\prime}, p \rightarrow 0\right)$

$$
\left(\frac{i}{a}\right)\left(\sin p_{\mu}-\sin p_{\mu}^{\prime}\right) \Lambda_{\mu L L}^{(1)}\left(p, p^{\prime}\right)=S_{L}^{-1}(p)-S_{L}^{-1}\left(p^{\prime}\right)
$$

where $S_{L}^{-1}(p)$ is the propagator of the left-handed chiral fermion and the Ward identity is realized by the chiral spectrum. Here we stress again that the disappearance of the three-fermion (right-handed) state at the threshold $\epsilon$ is essential point to obtain the continuum chiral gauge coupling (24) in the low-energy limit. However, we have to confess that similar to the threshold (16), eq. (39) is a plausible speculation for the time being, since we need more evidences and computations to show whether or not it is true.

4. Given the scenario of the chiral gauge coupling and spectrum (vector-like for $p \simeq \pi_{A}$ and chiral for $p \simeq 0$ ), one should expect that the gauge field should not only chirally couple to the massless chiral fermion of the $\psi_{L}^{i}$ in the low-energy regime, but also vectorially couple to the massive doublers of Dirac fermion $\Psi_{c}^{i}$ in the high-energy regime. We discuss the gauge anomaly and the renormalization of perturbative gauge theories.

We consider the following $n$-point 1PI functional:

$$
\Gamma_{\{\mu\}}^{(n)}=\frac{\delta^{(n)} \Gamma\left(A^{\prime}\right)}{\delta A_{\mu_{1}}^{\prime}\left(x_{1}\right) \cdots \delta A_{\mu_{j}}^{\prime}\left(x_{j}\right) \cdots \delta A_{\mu_{n}}^{\prime}\left(x_{n}\right)},
$$

where $j=1 \cdots n,(n \geq 2)$ and $\Gamma\left(A^{\prime}\right)$ is the vacuum functional. The perturbative computation of the 1PI vertex functions $\Gamma_{\{\mu\}}^{(n)}$ can be straightforwardly performed by adopting the method presented in ref. [1] for lattice QCD. Dividing the integration of internal momenta into 16 hypercubes where the 16 modes live, we have 16 contributions to the truncated vertex functions. The region where the chiral fermion modes of the continuum limit exist, is defined as

$$
\Omega=[0, \epsilon]^{4}, \quad p<\epsilon \ll \frac{\pi}{2}, \quad p \rightarrow 0,
$$

where $\epsilon$ is the energy-threshold given by (16), on which $\Psi_{R}(x)$ disappears.

As a first example, we deal with the vacuum polarization

$$
\Pi_{\mu \nu}(p)=\sum_{i=1}^{16} \Pi_{\mu \nu}^{i}(p), \quad \Pi_{\mu \nu}^{d}(p)=\sum_{i=2}^{16} \Pi_{\mu \nu}^{i}(p) .
$$

For the contributions $\Pi_{\mu \nu}^{d}(p)$ from the 15 doublers $(i=2, \ldots, 16)$, we make a Taylor expansion in terms of external physical momenta $p=\tilde{p}$ and the following 
equation is, mutatis mutandis, valid [1],

$$
\begin{aligned}
\Pi_{\mu \nu}^{d}(p) & =\Pi_{\mu \nu}^{\circ}(0)+\Pi_{\mu \nu}^{d(2)}(p)\left(\delta_{\mu \nu} p^{2}-p_{\mu} p_{\nu}\right) \\
& +\sum_{i=2}^{16}\left(1-\left.p_{\rho}\right|_{\diamond} \partial_{\rho}-\left.\frac{1}{2} p_{\rho} p_{\sigma}\right|_{\diamond} \partial_{\rho} \partial_{\sigma}\right) \Pi_{\mu \nu}^{c o n}\left(p, m_{i}\right),
\end{aligned}
$$

where $\left.\right|_{\circ} f(p)=f(0)$ and $m^{i}$ are doubler masses. The first and second terms are specific for the lattice regularization. Since the 15 doublers are gauged as an $S U(2)$ QCD-like gauge theory with propagator (6) and interacting vertex (37), the Ward identities (38) associated with this vectorial gauge symmetry result in the vanishing of the first divergent term $\Pi_{\mu \nu}^{\circ}(0)$ and the gauge invariance of the second finite contact term in eq.(43). We recall that in the Rome approach, this was achieved by enforcing Ward identities and gauge-variant counterterms. The third term in eq. (43) corresponds to the relativistic contribution of the 15 doublers. The $\Pi_{\mu \nu}^{c o n}\left(p, m_{i}\right)$ is logarithmically divergent and evaluated in some continuum regularization. For doubler masses $m_{i}$ of $O\left(a^{-1}\right)$, the third term in eq.(43) is just finite and gauge-invariant.

We turn to the contribution $\Pi_{\mu \nu}^{n}(p)$ of the normal mode that is in the first hypercube $\Omega=[-\epsilon, \epsilon]^{4}$ (41). Based on the plausible speculation that the normal mode and gauge coupling are chiral, we can use some regularization to calculate this contribution,

$$
\Pi_{\mu \nu}^{n}(p)=\Pi_{\mu \nu}^{n(2)}(p)\left(\delta_{\mu \nu} p^{2}-p_{\mu} p_{\nu}\right)
$$

The Ward identity (39) associated with this chiral gauge symmetry render eq. (44) gauge invariant. The $\epsilon$-dependence $(\ell n \epsilon)$ in eq. (44) has to be exactly cancelled by those contributions (43) from doublers, because of the continuity of 1PI vertex functions in momentum space. In summary, the total vacuum polarization $\Pi_{\mu \nu}(p)$ contains two parts: (i) the vacuum polarization of the normal mode (chiral) of $\psi_{L}^{i}$ in some continuum regularization; (ii) gauge invariant finite terms stemming from doubler contributions. The second part is the same as perturbative lattice QCD, and can be subtracted away in a normal renormalization prescription.

The second example is the 1PI vertex functional $\Gamma_{\{\mu\}}^{(n)}(\{p\})(n \geq 4)$,

$$
\begin{aligned}
\Gamma_{\{\mu\}}^{(n)}(\{p\}) & =\sum_{i=1}^{16} \Gamma_{\{\mu\}}^{(n) i}\left(\{p\}, m_{i}\right) \quad n \geq 4 \\
\{p\} & =p_{1}, p_{2}, \cdots \\
\{\mu\} & =\mu_{1}, \mu_{2}, \cdots,
\end{aligned}
$$

where the internal momentum integral is analogously divided into the contributions from sixteen sub-regions of the Brillouin zone where the sixteen modes live. Based on gauge invariance and power counting, one concludes that up to some gauge invariant finite terms, the $\Gamma_{\{\mu\}}^{(n)}(\{p\})(n \geq 4)$ (45) contain the 15 continuum expressions for 15 massive $\left(m_{i}\right)$ Dirac doublers and one for the massless chiral mode. The 15 doubler contributions vanish for $m_{i} \sim O\left(a^{-1}\right)$. The 
$n$-point 1PI vertex functions (45) end up with their continuum counterpart for the chiral fermion and some gauge invariant finite terms. These gauge invariant finite terms, which come from doubler contributions, are similar to those in Wilson lattice QCD, and can be subtracted away in the normal renormalization prescription.

The most important contribution to the vacuum functional is the triangle graph $\Gamma_{\mu \nu \alpha}(p, q)$, which is linearly divergent. Again, dividing the integration of internal momenta into 16 hypercubes, one obtains 11]

$$
\begin{aligned}
\Gamma_{\mu \nu \alpha}(p, q) & =\sum_{i=1}^{16} \Gamma_{\mu \nu \alpha}^{i}(p, q) \\
\Gamma_{\mu \nu \alpha}^{i}(p, q) & =\Gamma_{\mu \nu \alpha}^{i(\circ)}(0)+p_{\rho} \Gamma_{\mu \nu \alpha, \rho}^{i(1)}(0)+q_{\rho} \Gamma_{\mu \nu \alpha, \rho}^{i(1)}(0) \\
& +\left(1-\left.\right|_{\circ}-\left.p_{\rho}\right|_{\circ} \partial_{\rho}-\left.q_{\rho}\right|_{\circ} \partial_{\rho}\right) \Gamma_{\mu \nu \alpha}^{c o n}\left(p, q, m_{i}\right),
\end{aligned}
$$

where $\Gamma_{\mu \nu \alpha}^{c o n}\left(p, q, m_{i}\right)$ is evaluated in some continuum regularization. As for the 15 contributions of Dirac doublers $(i=2 \cdots 15)$, the first three terms in eq. 46) are zero owing to the vector-like Ward identity (38). The non-vanishing contributions are similar to those in Wilson lattice QCD. These contributions are gauge-invariant and finite (as $m_{i} \sim O\left(a^{-1}\right)$ ), thus, disassociate from the gauge anomaly.

The non-trivial contribution of the chiral mode in the hypercube $\Omega=[-\epsilon, \epsilon]^{4}$ is given by

$$
\begin{aligned}
\Gamma_{\mu \nu \alpha}^{i=1}(p, q)= & \int_{\Omega} \frac{d^{4} k}{(2 \pi)^{4}} \operatorname{tr}\left[S\left(k+\frac{p}{2}\right) \Gamma_{\mu}(k) S\left(k-\frac{p}{2}\right) \Gamma_{\nu}\left(k-\frac{p+q}{2}\right) S\left(k-\frac{p}{2}-q\right) \Gamma_{\alpha}\left(k-\frac{q}{2}\right)\right] \\
& +(\nu \leftrightarrow \alpha),
\end{aligned}
$$

where $S(p)=S_{L}(p)$ is the propagator of the left-handed chiral fermion, and vertex $\Gamma_{\mu}$ is given by eq.(24). Other contributions containing anomalous vertices $(\psi \bar{\psi} A A, \psi \bar{\psi} A A A)$ vanish within the hypercube $\Omega=[-\epsilon, \epsilon]^{4}$. As is well known, eq. (47) is not gauge invariant. To evaluate eq.(47), one can use some continuum regularization. As a result, modulo possible local counterterms, we obtain the consistent gauge anomaly for the non-abelian chiral gauge theories as the continuum one:

$$
\delta_{g} \Gamma\left(A^{\prime}\right)=-\frac{i g^{2}}{24 \pi^{2}} \int d^{4} x \epsilon^{\alpha \beta \mu \nu} \operatorname{tr} \theta_{a}(x) \tau_{a} \partial_{\nu}\left[A_{\alpha}(x)\left(\partial_{\beta} A_{\mu}+\frac{i g}{2} A_{\beta}(x) A_{\mu}(x)\right)\right],
$$

where the gauge field $A_{\mu}=\frac{\tau^{a}}{2} A_{\mu}^{a}$. The $S U_{L}(2)$ chiral gauge theory is anomaly-free for $\operatorname{tr}\left(\tau^{a},\left\{\tau^{b}, \tau^{c}\right\}\right)=0$, and the gauge current

$$
J_{\mu}^{a}=i \bar{\psi}_{L} \gamma^{\mu} \frac{\tau^{a}}{2} \psi_{L}=\frac{\delta \Gamma(A)}{\delta A_{\mu}^{a}(x)}, \quad \partial^{\mu} J_{\mu}^{a}=0
$$


is covariantly conserved and gauge invariant. It is surprising that we can consistently obtain the correct chiral gauge anomaly (48) in a speculative scenario, where the normal mode is chiral and doublers are massive and vector-like. What is interesting is that in this gauge invariant scenario and action (1)), we consistently achieve the chiral gauge anomaly, since this is different from the general idea that the origin of chiral gauge anomalies is due to the explicit breaking of chiral gauge symmetries at the cutoff. In the following paragraph, we give some discussion on this point.

A most subtle property of the naive lattice chiral gauge theory is the appearance of 16 modes. Each mode produces the chiral gauge anomaly with definite axial charge $Q_{5}$ [11], such that the finite (regularized) theory is anomaly-free and the chiral gauge symmetry is perfectly preserved. As has been seen, the 15 doublers are decoupled as massive Dirac fermions that are vector-gauge-symmetric (38). Thus, they decouple from the chiral gauge anomaly as well. Only the anomaly associated with the normal (chiral) mode of the $\psi_{L}^{i}$ is left and is the same as the continuum one. The condition for this circumstance to occur is the disappearance of the right-handed three-fermion state $\Psi_{R}^{i}$ in the low-energy limit. Otherwise, the chiral gauge anomaly (48) from the normal mode of $\psi_{L}^{i}$ would be exactly cancelled by that from $\Psi_{R}^{i}$. In future work, we will proceed to more detailed discussion on this point by looking at how this three-fermion state flows, dissolves into its constituents and fills up the Dirac sea[12].

It seems surprising that we start from a gauge symmetric action and end up with the correct gauge anomaly. Normally, one may claim that the anomaly has to come from the explicit breaking of the chiral gauge symmetry in a regularized action (e.g., the Wilson term). This statement is indeed correct if regularized actions are bilinear in fermion fields, since this is nothing but what the "no-go" theorem asserts. However, we run into the dilemma that the chiral gauge anomaly is independent of any explicit breaking parameters (e.g., the Wilson parameter $r$ and fermion masses). In fact, the most essential and intrinsic raison d'être of producing the correct chiral gauge anomaly is "decoupling doublers" rather than "explicit breaking of chiral gauge symmetries". If we adopt a bilinear action (e.g., the Wilson action) to decouple doublers, we must explicitly break chiral gauge symmetry, which is just a superficial artifact in bilinear actions. However, if we give up the bilinearity of regularized actions in fermion fields and find a chiral-gauge-invariant way (as the scenario we discussed) to decouple doublers, we should not be surprised to achieve the correct gauge anomaly (48).

The Ward identities (38) and (39) play an extremely important role to guarantee that the gauge perturbation theory in the scaling region $\left(1 \ll g_{2}<\infty\right)$ is gauge symmetric. To all orders of the gauge coupling perturbation theory, gauge boson masses vanish and the gauge boson propagator is gauge-invariantly transverse. The gauge perturbation theory can be described in the normal renor-

\footnotetext{
${ }^{1}$ This means no multifermion couplings (Yukawa couplings) and only gauge couplings.
} 
malization prescription as that of the QCD and QED theory. In fact, due to the manifest $S U_{L}(2)$ chiral gauge symmetry and corresponding Ward identities that are respected by the spectrum (vector-spectrum for $p \neq 0$ and chiral-spectrum for $p=0$ ) in this possible scaling regime, we should then apply the Rome approach [13] (which is based on the conventional wisdom of quantum field theories) to perturbation theory in the small gauge coupling. It is expected that the Rome approach should work in the same way but all gauge-variant counterterms are prohibited.

However, we cannot even speculate anything about the aspect of non-perturbative dynamics of the $S U_{L}(2)$ chiral gauge theory in this scaling segment, since the whole computation is strictly perturbative in the gauge coupling. The anomalous global symmetries are not involved in this paper, and readers are referred to the ref. 14.

I thank Profs. G. Preparata, M. Creutz, H.B. Nielsen and E. Eichten for discussions, and P. Ratcliffe for reading the manuscript.

\section{References}

[1] H.B. Nielsen and M. Ninomiya, Nucl. Phys. B185 (1981) 20, ibid. B193 (1981) 173, Phys. Lett. B105 (1981) 219.

[2] E. Eichten and J. Preskill, Nucl. Phys. B268 (1986) 179.

[3] Y. Nambu and G. Jona-Lasinio, Phys. Rev. 122 (1961) 345.

[4] M.F.L. Golterman, D.N. Petcher and E. Rivas, Nucl. Phys. B395 (1993) 597.

[5] J. Smit, Acta Physica Polonica B17 (1986) 531;

P.D.V. Swift, Phys. Lett. B145 (1984) 256.

[6] D.N. Petcher, Nucl. Phys.(Proc. Suppl.) B30 (1993) 52, references there in.

[7] S.-S. Xue, Phys. Lett. B381 (1996) 277 and Nucl. Phys. B486 (1997) 282.

[8] M.F.L. Golterman, D.N. Petcher, Phys. Lett. B225 (1989) 159.

[9] Y. Shamir, Phys. Rev. Lett. 71 (1993) 2691; Nucl. Phys.(Proc. Suppl.) B47 (1996) 212.

[10] S.-S. Xue, Nucl. Phys. (Proc. Suppl.) B53 (1997) 668.

[11] L.H. Karsren and J. Smit, Nucl. Phys. B183 (1981) 103.

[12] H.B. Nielsen and M. Ninomiya, Int. J. of Mod. Phys. A6 (1991) 2913;

M. Creutz and I. Horváth, Phys. Rev. D50 (1994) 2297. 
[13] A. Borrelli, L. Maiani, G.C. Rossi, R. Sisto and M. Testa, Nucl. Phys. B333 (1990) 335; Phys. Lett. B221 (1989) 360;

L. Maiani, G.C. Rossi, and M. Testa, Phys. Lett. B261 (1991) 479; ibid B292 (1992) 397;

L. Maiani, Nucl. Phys. (Proc.Suppl.) B29 (1992) 33.

[14] M. Creutz, M. Tytgat, C. Rabbi and S.-S. Xue, hep-lat/9612017, Phys. Lett. $\mathbf{B}$ in press. 